\title{
Balkanologie
}

Balkanologie Revue d'études pluridisciplinaires

Vol. $16 n^{\circ} 1 \mid 2021$

Au-delà de la "route des Balkans ": mondes sociaux des circulations

\section{L'Union européenne : mode d'emploi nationaliste. Notes sur le différend bulgaro-macédonien actuel}

The European Union: Nationalist User Manual. Notes on the Current BulgarianMacedonian Dispute

Tchavdar Marinov

(2) OpenEdition

Journals

Édition électronique

URL : https://journals.openedition.org/balkanologie/3130

DOI : 10.4000/balkanologie.3130

ISSN : 1965-0582

Éditeur

Association française d'études sur les Balkans (Afebalk)

Référence électronique

Tchavdar Marinov, « L'Union européenne : mode d'emploi nationaliste. Notes sur le différend bulgaromacédonien actuel », Balkanologie [En ligne], Vol. 16 n 1 | 2021, mis en ligne le 01 juin 2021, consulté le 03 octobre 2021. URL : http://journals.openedition.org/balkanologie/3130 ; DOI : https://doi.org/ 10.4000/balkanologie.3130

Ce document a été généré automatiquement le 3 octobre 2021.

(c) Tous droits réservés 


\title{
L'Union européenne : mode d'emploi nationaliste. Notes sur le différend bulgaro-macédonien actuel
}

\author{
The European Union: Nationalist User Manual. Notes on the Current Bulgarian- \\ Macedonian Dispute
}

Tchavdar Marinov

En octobre 1970, une délégation yougoslave de haut rang est en visite à Sofia pour discuter avec ses collègues bulgares des questions d'intérêt bilatéral. Contrairement à ce que l'on peut imaginer, la conversation entre les leaders des deux partis communistes ne fut nullement consacrée à des problèmes d'actualité tels que la guerre froide et l'opposition globale entre blocs politiques. Les questions idéologiques, comme l'interprétation et l'avenir du marxisme, ne furent pas non plus au menu. La rencontre s'inscrivait dans une longue série de débats au cours desquels les dirigeants bulgares et yougoslaves s'empoignaient autour de l'histoire et de l'identité de la population slave de Macédoine, ainsi que de sa langue. Dès les années 1960, les dirigeants bulgares rejetèrent notamment l'existence d'une nation et d'une langue macédoniennes puisque, à leur avis et selon les scientifiques de leur pays, le territoire de la république yougoslave la plus méridionale était peuplé, dès le haut Moyen Âge, par une majorité bulgare. L'appartenance ethnique de personnalités de l'ère médiévale, du XIX et du début du $\mathrm{xx}^{\mathrm{e}}$ siècle était un objet de discorde. Le paradoxe d'un différend nationaliste entre représentants politiques, par définition «internationalistes», était trop saisissant pour ne pas être remarqué, notamment par le représentant yougoslave Veljko Vlahović. Il exprima ainsi sa crainte " qu'au bout de trente ou cinquante ans, on ne rigole de nous et de la façon dont nous traitions du Moyen Âge $»^{1}$. 


\section{La genèse du différend actuel}

2 Proclamée en septembre 1991, l'indépendance de la République de Macédoine suscita une deuxième vague de controverses qui empoisonnèrent ses relations avec Sofia tout au long des années 1990. La Bulgarie fut le premier pays à reconnaître le nouvel État balkanique, dans le seul but d'encourager son divorce avec la Serbie, cette dernière étant traditionnellement vue à Sofia comme l'ingénieur principal de l'identité nationale macédonienne. Les nouveaux responsables politiques anticommunistes à Sofia ne tardèrent pas à dénoncer à leur tour la nation et la langue macédoniennes comme des "produits artificiels» d'une "propagande antibulgare» yougoslave. À la suite d'un rapprochement temporaire initié à la veille du nouveau millénaire, les rapports bilatéraux entre Sofia et Skopje restèrent vulnérables. Des scandales médiatiques réguliers agitaient le monde politique et académique des deux pays. Toutefois, pendant les deux premières décennies de l'indépendance macédonienne, le ton politique bulgare par rapport à son voisin du sud-ouest restait officiellement bienveillant - en réalité, paternaliste et condescendant. Dans l'imaginaire politique bulgare, les Macédoniens sont infantilisés et privés de subjectivité. Traités comme des fils prodigues de la « mère Bulgarie », ils sont censés intérioriser la " vérité historique » au sujet de leur origine bulgare, les apparatchiks «serbophiles » de l'époque yougoslave ayant quitté la scène politique.

3 La métamorphose attendue ne se produisit pourtant jamais. Par conséquent, dès 2011 , la politique bulgare prit un caractère plus intransigeant, proche de celui de la Grèce dans la querelle gréco-macédonienne parallèle ${ }^{2}$. Membre de l'Union européenne (UE) depuis 2007, la Bulgarie se voyait déjà dans une position privilégiée. Sans surprise, donc, l'ancienne république yougoslave - aspirant, elle aussi, à l'adhésion à l'UE - fit de son mieux pour apaiser les relations conflictuelles avec ses voisins. En 2019, elle accepta même de changer son nom en faveur de Macédoine du Nord, à la suite de l'accord de Prespa qui mit fin à son différend avec la Grèce. Le différend bulgaromacédonien quant à lui éclata de nouveau la même année, étonnamment, après la signature d'un "Traité d'amitié, de bon voisinage et de coopération » (août 2017) par le Premier ministre bulgare Boyko Borisov et son homologue macédonien Zoran Zaev.

En vertu de ce traité, Sofia et Skopje créèrent une commission bilatérale d'experts (surtout des historiens) chargés de proposer des formules consensuelles concernant les points contentieux. Cependant, la tâche précise de la commission reste floue à ce jour, en raison des attentes divergentes des deux pays. Skopje confia à son équipe d'experts une mission raisonnable par son minimalisme: par exemple, proposer des recommandations pour la révision de manuels scolaires dans un esprit de bon voisinage. Côté bulgare, ce fut jugé insuffisant: dès le début, les spécialistes de la commission étaient appelés par les milieux dirigeants à Sofia à attester des «faits historiques ». Du point de vue des élites bulgares, les experts de Skopje devraient reconnaître publiquement les «racines» ethniques bulgares des Macédoniens, l'identité nationale bulgare des figures de leur panthéon national et la base dialectale bulgare de la langue macédonienne. En fait, le gouvernement de Borisov, une coalition de droite avec la participation du parti nationaliste VMRO-BND ${ }^{3}$ spécialisé dans la "question macédonienne", voulut utiliser la commission comme un levier pour imposer à la Macédoine du Nord les interprétations bulgares traditionnelles. En fin de compte, il proposa aux experts macédoniens et au gouvernement de Skopje un faux 
choix : accepter les «faits historiques" bulgares ou faire face à un veto bulgare bloquant la candidature du pays à l'adhésion à l'UE.

5 Résultat : le dialogue entre les deux équipes nationales au sein de la commission mixte s'avéra difficile, se muant par la suite en querelles médiatiques autour de l'appartenance nationale de différentes personnalités historiques. La " désobéissance » macédonienne ne fit qu'intensifier la stratégie bulgare décrite par des observateurs comme une politique de «brimade $»^{4}$. En octobre 2019, le gouvernement de Borisov adopta une «Position-cadre » et le parlement bulgare rendit publique une déclaration fustigeant le récit historique macédonien comme une falsification de l'histoire bulgare. Ces mêmes documents adressent à la Macédoine du Nord des demandes focalisées sur des questions identitaires comme conditions préalables à l'ouverture de négociations avec l'UE. Sofia envoya aux diplomates des autres États membres de l'UE un "Mémorandum explicatif» nationaliste présentant les thèses officielles bulgares comme des « faits historiques » attestés. Selon ce document, avant la fin de la Seconde Guerre mondiale, la population slave de Macédoine était bulgare et la nation macédonienne aurait été créée par les communistes yougoslaves au prix d'une terreur impitoyable. Le mémorandum fait état de 100000 victimes présumées du régime de Tito, tuées ou opprimées à cause de leur patriotisme bulgare - un nombre certainement fantaisiste.

6 Les menaces de veto bulgares se concrétisèrent finalement le 17 novembre 2020 lors d'une réunion du Conseil des affaires générales de l'UE, au cours de laquelle la ministre bulgare des Affaires étrangères, Ekaterina Zakharieva, déclara que Sofia désapprouvait le cadre de négociation avec la Macédoine du Nord et opposa donc son veto aux discussions avec Skopje qui devaient commencer en décembre dernier.

\section{Acteurs et stratagèmes politiques bulgares}

7 Dès le début des controverses, le Premier ministre bulgare Borisov préféra garder son image de bon élève européen, cher à la chancelière Merkel et aux fondations de la droite allemande, pour s'assurer l'accès aux généreux fonds européens. La définition et la mise en place de la politique bulgare par rapport à la Macédoine du Nord furent confiées à des acteurs relevant du ministère des Affaires étrangères, des services de renseignement et à des partenaires politiques de coalition, tels que le chef du VMROBND et ministre de la Défense Krasimir Karakatchanov. Les fers de lance de cette politique ne mâchaient pas leurs mots: Zakharieva et Karakatchanov insistèrent ouvertement, à plusieurs reprises, pour que l'élite politique macédonienne reconnaisse les «racines bulgares» des Macédoniens. Le comportement provocateur de Karakatchanov atteignit un sommet en mars 2021 lorsqu'il publia sur un réseau social une carte de vœux représentant les contours de la Grande Bulgarie de San Stefano (1878). Les députés bulgares au Parlement européen firent également preuve de zèle. Le représentant du VMRO-BND Angel Djambazki comparait la Macédoine du Nord avec la Corée du Nord devant ses collègues à Strasbourg et à Bruxelles. Andrey Kovatchev, député issu du parti de Borisov (GERB), ancien questeur du Parlement européen et biologiste de formation, ne cessa, dès son élection en 2009, d'exposer au sein du Parlement européen la « vérité historique » sur la Macédoine.

8 La surenchère nationaliste gagna les camps politiques de l'opposition en Bulgarie, en particulier celui du Président de la République Roumen Radev. À plusieurs occasions, 
celui-ci insinua que le gouvernement de Borisov était prêt à faire des compromis avec les "intérêts nationaux " bulgares, notamment pour garder son image de docilité devant les institutions européennes. Au-delà des calculs et des tactiques de mobilisation conjoncturelle, certes réels, un consensus nationaliste règne au sein des élites politiques en Bulgarie au sujet des controverses identitaires avec la Macédoine du Nord. Le gouvernement intérimaire, nommé par Radev à la suite des élections législatives en avril 2021, suit la même ligne d'intransigeance par rapport à Skopje. Le 22 juin 2021, il confirma le veto bulgare lors d'une nouvelle session du Conseil des affaires générales de l'UE.

Officiellement, Sofia reproche à Skopje de ne pas respecter le Traité d'amitié de 2017. Les responsables bulgares affirment notamment que la commission d'experts bilatérale n'a pas suffisamment progressé dans ses travaux en raison d'un manque de volonté, voire d'une hostilité, du côté macédonien. Le blocage bulgare des négociations avec Skopje rencontre néanmoins peu de sympathie auprès des institutions et des dirigeants européens : ces derniers ne semblent pas enthousiastes devant la perspective d'une nouvelle dispute nationaliste autour de la Macédoine. Les leaders du VMRO-BND à Sofia se plaignaient d'une « pression » occidentale en faveur de Skopje. À deux occasions, les ministres des Affaires étrangères tchèque et slovaque critiquèrent à haute voix la politique bulgare en déclarant que l'UE ne saurait être un arbitre de différends historiques nationalistes. En mars 2021, une réprimande du comportement provocateur d'officiels de Sofia, proposée par les socialistes au Parlement européen, fut formulée dans un rapport de ce dernier au sujet de la Macédoine du Nord, mais la critique fut supprimée au dernier moment en résultat des protestations bulgares.

Conscients du fait que leur rhétorique tombe dans l'oreille d'un sourd auprès des institutions de l'UE, les représentants bulgares mobilisent deux types d'argumentaires censés gagner la sympathie dans le contexte libéral dominant en Europe. D'une part, ils essaient d'instrumentaliser la politique mémorielle européenne au sujet des «régimes totalitaires » du $\mathrm{xx}^{\mathrm{e}}$ siècle. L'objectif de Sofia est notamment de diffamer l'identité nationale macédonienne en la présentant comme le produit de la Yougoslavie titiste, d'où les analogies avec la Corée du Nord suggérant que des structures secrètes, en place depuis l'époque communiste, dirigeraient toujours la scène politique de la Macédoine du Nord. Ces allégations peinent à convaincre. En 2021, la Bulgarie occupe, pour la quatrième année consécutive, la dernière place en Europe (et la $112^{\mathrm{e}}$ dans le monde) en matière de liberté des médias, derrière la Macédoine du Nord classée $90^{05}$. En réalité, l'argumentaire bulgare lui-même reprend, presque mot pour mot, des « thèses » et des « rappels historiques » concernant la langue slave et l'histoire de la Macédoine publiés sous le régime de Jivkov ${ }^{6}$. Les revendications adressées aux homologues politiques macédoniens sont également identiques, de la requête d'organiser des commémorations communes de personnalités et d'événements historiques (Cyrille et Méthode, l'insurrection d'Ilinden...) à la signature de documents officiels stipulant les «faits historiques». Les initiatives de ce genre sont présentées par Sofia comme des gestes de « bon voisinage » qui reconnaissent l'« histoire commune » (obchta istoriia en bulgare) des deux pays. Ce concept est cependant extrêmement idéologique : du côté bulgare, il sous-entend une reconnaissance par Skopje de l'« histoire bulgare » de la Macédoine.

11 D'autre part, essayant d'affiner la rhétorique héritée du régime communiste, les responsables bulgares la décorent de clichés libéraux. Par exemple, ils se plaignent 
régulièrement du «discours de haine " antibulgare en Macédoine du Nord. Certes, il existe à Skopje des milieux politiques, des commentateurs publics et des médias connus pour leurs sentiments antibulgares, mais ils ne représentent pas la ligne politique de l'actuel gouvernement. Les milieux politiques à Sofia se montrent particulièrement irrités par certains stéréotypes populaires touchant aux Bulgares, qui existent effectivement en Macédoine du Nord, et par la manière dont la période d'occupation bulgare du pays pendant la Second Guerre mondiale est présentée dans l'espace public et les manuels scolaires macédoniens. À toutes sortes d'occasions, le gouvernement macédonien est appelé par Sofia à réagir contre la "haine». Pourtant, on peut se demander dans quelle mesure le système scolaire bulgare encourage des images positives des voisins balkaniques, ou bien si le gouvernement de Sofia réagit contre tous les types de stéréotypes collectifs s'appuyant sur l'histoire. Les chaînes de télévision nationales ne cessent de diffuser, par exemple, le «film le plus aimé des Bulgares ", Temps de division (Vreme razdelno, 1988). Créé en pleine campagne de bulgarisation des noms de la minorité turque sous le régime de Jivkov, foisonnant de clichés antiturcs et d'un naturalisme brutal, ce film ne fait pas réagir les dirigeants du pays et, d'ailleurs, Ankara ne tient pas ces derniers pour responsables de ces diffusions.

12 La notion de « discours de haine » est utilisée par la Bulgarie de manière très arbitraire. Souvent, l'existence même d'une nation macédonienne semble être considérée par Sofia comme un acte antibulgare. Dans le même temps, contrairement au comportement diplomatique du gouvernement de Zaev, des représentants du gouvernement bulgare, tels que Karakatchanov, se permettaient d'utiliser une rhétorique brutale qui offense parfois non seulement la Macédoine du Nord mais aussi la Serbie. Des cas d'oppression, réelle ou présumée, exercée sur des individus natifs de Macédoine avec une conscience ethnique bulgare ou ayant la double nationalité macédonienne et bulgare, sont largement publicisés par les autorités de Sofia comme preuves du caractère insincère et agressif de Skopje. En réalité, le gouvernement de Borisov et le Président Radev ont fait de leur mieux pour alimenter des réactions publiques antibulgares en Macédoine du Nord. De plus, les institutions bulgares sont loin de faire preuve d'un décorum démocratique européen, notamment par leur refus constant d'enregistrer des partis prétendant représenter des Macédoniens ethniques en Bulgarie. À ce titre, Sofia a été condamnée à plusieurs reprises par la Cour européenne des droits de l'homme et critiquée par le Conseil de l'Europe -notamment en octobre 2020. Suivant le principe que l'attaque est la meilleure défense, les autorités bulgares ont commencé à fustiger Skopje pour avoir prétendument insisté sur l'existence d'une minorité macédonienne en Bulgarie. Or, la politique officielle macédonienne a abandonné de telles affirmations depuis des décennies.

\section{Experts et prises de position publiques}

13 Un bref regard sur l'espace public bulgare est suffisant pour constater que le consensus nationaliste sur la "question macédonienne" n'est nullement limité au champ politique. En règle générale, les médias s'empressent d'applaudir la ligne gouvernementale : l'expression d'avis alternatifs sur celle-ci est presque impossible sur les chaînes de télévision et les stations de radio nationales. Corée du Nord? À la différence de cette dernière, quelques sites internet au profil libéral publient des critiques, bien qu'elles restent souvent timides. Le consensus nationaliste est plus que 
visible dans le champ académique qui, par tradition, fournit servilement aux autorités politiques le récit dominant. La situation est similaire en Macédoine du Nord, à quelques différences près. En témoignent, par exemple, les positions plus progressistes de l'équipe d'experts macédoniens de la commission bilatérale bulgaro-macédonienne chargée de proposer des solutions aux questions sensibles.

14 L'État macédonien est en position de faiblesse par rapport à la Bulgarie. Dès lors, le gouvernement macédonien a intégré dans l'équipe plusieurs chercheurs relativement jeunes, dotés d'une formation universitaire internationale et d'un mode de pensée plus ou moins libéral. Il n'a pas fait appel à de grands académiciens macédoniens au profil nationaliste. Il est clair que l'expérience universitaire internationale ne fournit pas forcément de contrepoids aux doctrines établies du récit national. Par exemple, certains membres de l'équipe macédonienne ont instrumentalisé les théories constructivistes de l'identité nationale pour démontrer que la nation macédonienne a été un fait accompli au XIX ${ }^{\mathrm{e}}$ siècle, rejetant ainsi toute idée d'une " histoire commune " des Macédoniens et des Bulgares? ${ }^{7}$. Ils ont également critiqué ce qu'ils voyaient comme des concessions excessives à la Bulgarie de la part du Premier ministre Zaev. Mais d'autres experts macédoniens de la commission bilatérale ont appelé à une réflexion critique sur les «mythes » au sein du récit historique macédonien ${ }^{8}$, devenant les cibles d'accusations de «trahison nationale » lancées par des commentateurs médiatiques et par leurs propres collègues.

15 Côté bulgare, la position des experts ne laisse aucun dilemme ouvert. Les autorités bulgares ont formé une équipe d'historiens mainstream et de diplomates, souvent des personnes occupant des postes administratifs importants au sein d'institutions nationales. Leurs déclarations publiques laissent apparaitre l'idée qu'ils se font de leur rôle dans la commission, s'apparentant à des généraux de l'armée défendant une cause nationale ou à des inspecteurs de police censés faire reconnaître aux malfaiteurs leurs méfaits. Ces spécialistes bulgares manifestent un désintérêt total pour une reconsidération critique quelconque du récit canonique bulgare et une absence flagrante de compétence dans le domaine des études contemporaines de la construction des identités et des langues nationales. Qui plus est, leur comportement s'est éloigné à plusieurs reprises du ton politiquement neutre obligatoire dans des débats scientifiques. Les déclarations publiques de l'historien de la littérature Kiril Topalov, doyen de l'équipe bulgare, constituent un paroxysme de clichés et de sens détourné au sujet des «racines bulgares» des Macédoniens, un foisonnement d'attaques brutales adressées à l'identité macédonienne prétendument inculquée par le «totalitarisme féroce» dans la «Corée du Nord balkanique». Une naïveté méthodologique saisissante règne dans les propos des autres experts, tel Naoum Kaytchev, chef de la chaire d'histoire balkanique à l'Université de Sofia, diplomate et activiste du VMRO-BND'. Sans surprise, au bout d'une série de conversations difficiles consacrées à l'interprétation d'une longue liste de personnalités historiques rangées de manière chronologique, le travail de la commission bilatérale s'est fracassé contre la figure du révolutionnaire macédonien Gotse Deltchev (1872-1903). Par conséquent, les experts se sont retrouvés au point de départ et la commission, d'après l'expression d'un journal d'opposition bulgare, " a coulé dans le haut Moyen Âge ».

16 Certes, des voix alternatives existent dans les deux pays. Du côté macédonien, des figures telles que l'ancien ministre des Affaires étrangères Denko Maleski et la philosophe Katerina Kolozova ont provoqué à plusieurs reprises la colère du grand 
public avec des déclarations jugées "pro-bulgares ». Des accusations de ce genre n'ont pas été épargnées au Président de la République Stevo Pendarovski et au Premier ministre Zaev qui cherchent visiblement des formules de compromis. En Bulgarie, une série d'initiatives de rapprochement - entre autres, des conférences entre chercheurs et militants des deux pays et des lettres ouvertes adressées aux autorités bulgares et macédoniennes - ont été lancées par des intellectuels libéraux, dont le plus actif est l'historien Stefan Detchev ${ }^{10}$.

17 Cependant, cette activité est restée plutôt dans les marges de l'espace public et du champ académique. Pour ce qui est de la Bulgarie, la plupart des historiens d'expérience internationale et au profil libéral évitent de traiter de sujets liés à la Macédoine, la "question macédonienne " étant à leurs yeux une problématique surchargée de primitivisme nationaliste. Mais, par contrecoup, cette absence d'intérêt empêche le développement d'une réflexion critique sur les questions contentieuses. De ce point de vue, la réaction de Maria Todorova, la plus grande autorité d'origine bulgare dans le champ international des études historiques, était symptomatique: Todorova a préféré commenter le différend actuel par des anecdotes ${ }^{11}$.

De plus, une bonne partie des publications bulgares dirigées contre la ligne officielle sont marquées de stéréotypes nationalistes. Souvent, le souci principal des auteurs est que le manque de tact par rapport à Skopje repousse « nos frères macédoniens » et sert des intérêts géopolitiques étrangers - russes, voire serbes. Tout en critiquant le conservatisme de leurs collègues, les historiens libéraux entrent parfois dans la position de mentors, convaincus qu'il est légitime d'éclairer non seulement les Bulgares mais aussi les Macédoniens sur ce qu'ils sont censés croire à propos de leur histoire une attitude paternaliste dont ils ne se serviraient pas par rapport aux Turcs, aux Grecs ou aux Roumains. Cette position laisse voir une perception sous-jacente des Macédoniens qui réitère leur image de Bulgares désorientés, dominante en Bulgarie. Il serait donc simpliste de réduire le débat à une confrontation entre conservateurs nationalistes et libéraux éclairés.

Nous ne pouvons faire qu'un constat plutôt triste. Cinquante ans après la rencontre au sommet à Sofia entre dirigeants communistes bulgares et yougoslaves mentionnée en introduction, à bien des égards, le contenu des débats politiques, publics et historiographiques n'a pas changé. Contrairement à la remarque du Monténégrin Vlahović, il n'y a donc pas beaucoup de raisons de rigoler en Bulgarie. Encore moins en Macédoine ex-yougoslave.

\section{NOTES}

1. AMVnR (Archive du ministère des Affaires étrangères bulgare) /27/158/3258/117

2. Le différend entre la Grèce et la république ex-yougoslave concernait principalement la désignation "Macédoine ", utilisée par cette dernière mais également portée par une région en Grèce du Nord autour de Thessalonique: d'où les accusations d'irrédentisme supposé par Athènes, fondées également sur certaines revendications initiales, de la part de Skopje, 
concernant l'existence d'une "minorité ethnique macédonienne " dans le nord de la Grèce. L'autre point de contention était la référence au patrimoine historique de la Macédoine ancienne dans le récit historique macédonien, laquelle fut très accentuée par le régime de Nikola Gruevski entre 2006 et 2016. Athènes, pourtant, considère l'État antique de Philippe et d'Alexandre le Grand comme sa propriété symbolique.

3. Vatrechna makedonska revoliutsionna organizatsiia - Balgarsko natsionalno dvijenie [Organisation révolutionnaire intérieure macédonienne - Mouvement national bulgare].

4. BIEBER Florian, « How Bulgarian Bullying Against North Macedonia Threatens EU Enlargement, Again ", European Western Balkans, 3 novembre 2020, en ligne: https:// europeanwesternbalkans.com/2020/11/03/how-bulgarian-bullying-against-north-macedoniathreatens-eu-enlargement-again/ (consulté en juin 2021).

5. Selon l'organisation Reporters sans frontières, en ligne: https://rsf.org/fr/classement (consulté en juin 2021).

6. Sur les controverses identitaires entre la Bulgarie et la Yougoslavie/la Macédoine yougoslave à l'époque socialiste, voir: TROEBST Stefan, Die bulgarisch-jugoslawische Kontroverse um Makedonien 1967-1982 [La controverse bulgaro-yougoslave sur la Macédoine 1967-1982], Munich, oldenbourg, 1983 ; MARINOv Tchavdar, La question macédonienne de 1944 à nos jours. Communisme et nationalisme dans les Balkans, Paris, L'Harmattan, 2010.

7. SPASKOVSKA Ljubica, "Site nacii se moderni konstrukti» [Toutes les nations sont des constructions modernes], ResPublica, $1^{\text {er }}$ août 2019, en ligne: https://respublica.edu.mk/mk/ blog/2019-08-01-10-48-19 (consulté en juin 2021).

8. GJORGIEV Dragi, TODOROV Petar, « Makedonsko-bugarskiot spor ne može da ima "pobednik" » [Il ne peut pas y avoir de «vainqueur » dans la querelle macédo-bulgare], Deutsche Welle, 25 avril 2020, en ligne : https://p.dw.com/p/3bNhD (consulté en juin 2021).

9. Kaytchev est convaincu que les sources historiques parlent elles-mêmes en faveur du point de vue bulgare : "Razkovnitcheto na recheniiata mejdu Sofia i Skopje ne e v otstapki, a v izvorite " [La clé des solutions entre Sofia et Skopje n'est pas dans les concessions mais dans les sources], Dnevnik, 14 octobre 2020, en ligne: https://www.dnevnik.bg/sviat/ 2020/10/14/4126482_doc_naum_kaichev_razkovnicheto_na_resheniiata_mejdu/ (consulté en juin 2021).

10. Voir par exemple « Otvoreno pismo ot balgarski i makedonski utcheni: Na karta e postaveno priiatelstvoto i dobrosasedstvoto » [Lettre ouverte de chercheurs bulgares et macédoniens : jouer la carte de l'amitié et du bon voisinage], Marginalia, 3 novembre 2020, en ligne: https:// www.marginalia.bg/fokus/otvoreno-pismo-ot-balgarski-i-makedonski-ucheni-na-karta-epostaveno-priyatelstvoto-i-dobrosasedstvot/ (consulté en juin 2021).

11. TODorova Maria, "On Happiness and Nationalism », Balkan Insight, 3 juin 2020, en ligne : https://balkaninsight.com/2020/06/03/on-happiness-and-nationalism/ (consulté en juin 2021).

\section{AUTEUR}

\section{TCHAVDAR MARINOV}

IPHS, Académie des sciences bulgare

tchmarinov[at]gmail.com 